\title{
Collisional dissipation rate in shearing flows of granular liquid crystals
}

\author{
Diego Berzi, ${ }^{1}$ Nha Thai-Quang, ${ }^{2}$ Yu Guo, ${ }^{2}$ and Jennifer Curtis ${ }^{3}$ \\ ${ }^{1}$ Dipartimento di Ingegneria Civile e Ambientale, Politecnico di Milano, 20133 Milano, Italy \\ ${ }^{2}$ Department of Chemical Engineering, University of Florida, Gainesville, Florida 32611, USA \\ ${ }^{3}$ College of Engineering, University of California Davis, Davis, California 95616, USA
}

(Received 10 March 2017; published 24 May 2017)

\begin{abstract}
We make use of discrete-element-method numerical simulations of inelastic frictionless cylinders in simple shearing at different length-to-diameter ratios and solid volume fractions to analyze the rate of collisional dissipation of the fluctuation kinetic energy. We show that the nonspherical geometry of the particles is responsible, by inducing rotation, for increasing the dissipation rate of the fluctuation kinetic energy with respect to that for frictionless spheres. We also suggest that the partial alignment of the cylinders induced by shearing concurs with the particle inelasticity in generating correlation in the velocity fluctuations and thus affecting the collisional dissipation rate as the solid volume fraction increases. Finally, we propose simple phenomenological modifications to the expression of the collisional dissipation rate of kinetic theory of granular gases to take into account our findings.
\end{abstract}

DOI: 10.1103/PhysRevE.95.050901

\section{INTRODUCTION}

Predicting the flow dynamics of nonspherical, nonBrownian particles is of paramount importance in many industrial and geophysical applications. As in the case of granular flows composed of spheres, models based on the solution of the equations of motion for the individual particles that take into account in a sophisticated way the particleparticle interactions are now available for cylinders [1-4]. It has been demonstrated that these models are in qualitative and quantitative agreement with some measurements on flows of glass cylinders and wooden pegs in split bottom shear cells [5] and gravity-driven collisional suspensions of plastic cylinders in water $[6,7]$. However, the discrete-element simulations are computationally demanding and their application is therefore limited to flows involving a few thousand particles. Flows at larger scale would inevitably require a continuum modeling approach, which is still lacking.

Recently [8], we proposed to adopt an approach based on kinetic theory of granular gases $[9,10]$ when dealing with cylindrical particles. When compared with the measurements in discrete-element simulations of simple shearing of frictionless cylinders of different length-to-diameter (aspect) ratio, the particle pressure and shear stress were well predicted by kinetic theory, provided that the solid volume fraction at which the stresses are singular was adjusted; the expression for the shear stress of kinetic theory was multiplied by a factor associated with the degree of order in the particle alignment. We have indeed shown that frictionless cylinders in simple shearing are in general in a liquid crystal phase, i.e., spatially disordered and orientationally, partially ordered. We have also suggested that the degree of order results from the balance between the alignment induced by the shearing and the randomization due to the velocity fluctuations and provided a simple expression for it.

Given the key role played by velocity fluctuations in kinetic theory, the simplest continuum theory for nonspherical particles based on it must include a differential equation governing the evolution of a measure of those fluctuations (balance of fluctuation kinetic energy [9]). In that balance, fluctuation kinetic energy is dissipated due to the inelastic nature of collisions and a constitutive relation for the rate of dissipation must be provided.

Here we focus on obtaining an expression for the collisional dissipation rate for frictionless cylinders by making use of discrete-element simulations of simple shearing flows. We investigate the role played by the nonspherical geometry of the particles and the particle alignment and how to modify, accordingly, the expression of kinetic theory. In this respect, we also want to check if the collisional dissipation rate predicted by classic kinetic theory overestimates the measurements at large solid volume fractions, as is the case for spheres [11,12]. That observation, explained by a decrease of the collision frequency associated with the development of correlation in the particle velocities (i.e., the breaking of the molecular chaos assumption), led to the proposal of an extended version of kinetic theory, which includes a correlation length in the expression of the collisional dissipation rate, and has been already tested in a number of flow configurations [13-17].

\section{NUMERICAL SIMULATIONS AND PREVIOUS RESULTS}

We have carried out numerical simulations of simple shearing of frictionless cylinders of mass density $\rho_{p}$, length $l$, and diameter $d$ with the discrete-element model described in Refs. $[1,3,4]$. We take $x$ to be the direction of the mean particle flow and use Lees-Edwards [18] boundary conditions along $y$ (perpendicular to $x$ ), resulting in a linear distribution of the mean particle velocity along this direction. The direction $z$, perpendicular to both $x$ and $y$, is the direction of the vorticity. Periodic boundary conditions have been employed along $x$ and $z$, to ensure uniformity of the flows along these directions. The ratio $l / d$ represents the cylinder aspect ratio. In what follows, we indicate as flat (elongated) cylinders particles for which $l / d$ is less (greater) than one.

We have performed the simulations by keeping constant the normal coefficient of collisional restitution (the negative 
of the ratio of post- to precollisional relative velocity between two colliding particles) $e=0.95$; the dimensionless Young modulus $E /\left(\rho_{p} \dot{\gamma}^{2} d_{v}^{2}\right)=1.5 \times 10^{9}$, where $d_{v}=\left(3 d^{2} l / 2\right)^{1 / 3}$ is the diameter of the equivalent sphere; and the Poisson ratio equal to 0.3 in the Hertzian contact model. We then imposed the shear rate $\dot{\gamma}$, the solid volume fraction $v$, and the aspect ratio of the particles and measured the steady-state values of the particle pressure $p$, shear stress $s$, granular temperature $T$ (mean of the square particle velocity fluctuations, i.e., a measure of the strength of the fluctuations in the translational velocity), and order parameter $S .{ }^{1}$ The latter describes the degree of order in the particle alignment: We define as granular gases, granular solid crystals, and granular liquid crystals particulate systems for which $S=0, S=1$, and $0<S<1$, respectively. We have changed the particle aspect ratio $l / d$ in the range $0.125-8$ and the solid volume fraction $v$ in the range 0.025-0.67. Examples of the flow configuration for flat and elongated cylinders at solid volume fraction $v=0.4$ are depicted in Fig. 1.

In our previous study [8] we showed that the particle pressure can be well predicted, irrespective of the particle aspect ratio and for the whole range of volume fraction, using the constitutive relation of kinetic theory of granular gases obtained for randomly colliding spheres [10],

$$
p=\rho_{p}\left[v+2(1+e) v^{2} g_{0}\right] T,
$$

where $g_{0}$ is the radial distribution function at contact. For the latter, we used the expression suggested by Vescovi et al. [17] and valid for frictionless spheres with $e$ less than or equal to 0.95 :

$$
g_{0}=\frac{2-v}{2(1-v)^{3}}
$$

if $v \leqslant 0.4$ and

$g_{0}=\left[1-\left(\frac{v-0.4}{v_{c}-0.4}\right)^{2}\right] \frac{2-v}{2(1-v)^{3}}+\left(\frac{v-0.4}{v_{c}-0.4}\right)^{2} \frac{2}{v_{c}-v}$

if $v>0.4$. In Eq. (3), $v_{c}$ represents the critical value of the solid volume fraction at which $g_{0}$ is singular for perfectly rigid spheres, which is only a function of the surface friction for spheres [19]. In the case of frictionless cylinders, $v_{c}=0.67$ allows good agreement with the data [8].

While the particle pressure is not substantially affected by the anisotropy induced by the particle alignment (given the independence of the particle aspect ratio), the particle shear stress is strongly sensitive to the orientational order. For frictionless cylinders, we have shown [8] that the expression of kinetic theory of granular gases [10] still gives a good estimate of the particle shear stress in granular liquid crystals provided it is multiplied by $1-S$ :

$$
s=(1-S) \rho_{p} \frac{8 J}{5 \pi^{1 / 2}} v^{2} g_{0} d_{v} T^{1 / 2} \dot{\gamma},
$$

\footnotetext{
${ }^{1}$ As for molecular liquid crystals, we calculate $S$ as the largest eigenvalue of the symmetric traceless tensor $Q_{i j}=3 /(2 N) \sum_{n-1}^{N}\left(\ell_{i}^{n} \ell_{j}^{n}-\right.$ $\left.\delta_{i j} / 3\right)$, where $\ell^{n}$ is the unit vector along the axis of the $n$th cylindrical particle and the sum is over all the $N$ particles of the system [5].
}

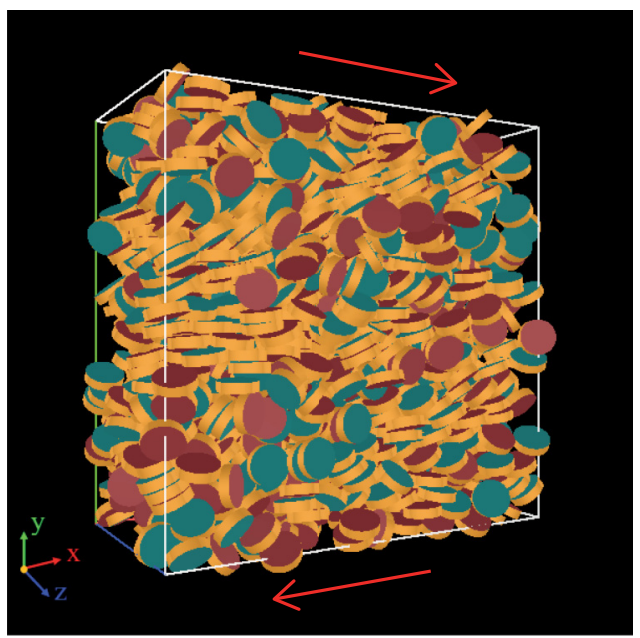

(a)

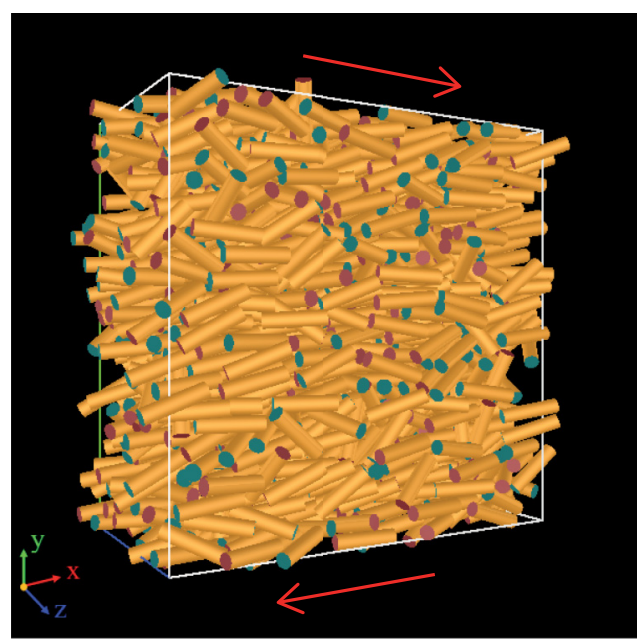

(b)

FIG. 1. Snapshots of the shear flows of (a) flat $(l / d=0.25)$ and (b) elongated $(l / d=4)$ cylinders at $v=0.4$.

where $J$ is a known function of the coefficient of restitution and the solid volume fraction. ${ }^{2}$ The rationale behind Eq. (4) is that the particle shear stress of a granular liquid crystal can be decomposed into two components, associated with the orientationally random and ordered motion of the particles, respectively proportional to $1-S$ and $S$. Neglecting the contribution due to the orientationally ordered motion (a seemingly reasonable assumption for frictionless cylinders) and assuming that the contribution of the orientationally random motion obeys the expression of kinetic theory results in Eq. (4).

We have also suggested [8] that the order parameter in Eq. (4) should be an increasing function of $d_{v} \dot{\gamma} / T^{1 / 2}$, given that shearing and velocity fluctuations favor alignment and randomization, respectively. The numerical simulations [8]

\footnotetext{
${ }^{2}$ Here $\quad J=(1+e) / 2+(\pi / 32)\left[5+2(1+e)(3 e-1) v g_{0}\right][5+4$ $\left.(1+e) v g_{0}\right] /\left[24-6(1-e)^{2}-5\left(1-e^{2}\right)\right] v^{2} g_{0}^{2}$, from, e.g., Jenkins and Berzi [14].
} 
confirmed this idea and gave

$$
S=\frac{1}{1+\exp \left(6-4 \dot{\gamma} d_{v} / T^{1 / 2}\right)} .
$$

The actual form of Eq. (5) is not crucial and other expressions are certainly possible. The key point is that Eqs. (1) and (4), with Eq. (5), relate the particle stresses to the solid volume fraction, the particle mean velocity (through the shear rate), and the granular temperature which represent, therefore, the hydrodynamic fields in a possible continuum model, based on kinetic theory, for the motion of granular liquid crystals. These hydrodynamic fields are the solutions to the differential equations expressing the balances of mass, momentum, and fluctuation kinetic energy. In the latter, constitutive relations for the collisional dissipation rate and for the energy flux, whose divergence represents the energy diffusion due to random motion, are needed to close the differential problem.

\section{COLLISIONAL DISSIPATION RATE AND CORRELATION LENGTH}

In simple shearing, the granular temperature and the volume fraction are uniform throughout the flow, so the fluctuation energy flux, which is proportional to gradients in $T$ and $v$ [10], vanishes. The fluctuation (translational) kinetic energy balance for inelastic frictionless spheres reduces, therefore, to a balance between the energy produced through the work of the shear stress and the energy dissipated in collisions:

$$
s \dot{\gamma}=\Gamma,
$$

where $\Gamma$ is the collisional dissipation rate. In the absence of velocity correlation among the particles (i.e., when the molecular chaos assumption holds; for spheres, this is the case when $v \leqslant 0.49[11,16])$, the expression of kinetic theory [10] for the collisional dissipation rate reads

$$
\Gamma=\rho_{p} \frac{12}{\pi^{1 / 2}} v^{2} g_{0}\left(1-e^{2}\right) \frac{T^{3 / 2}}{d_{v}} .
$$

Equation (7) with $e=0.95$ and $v \leqslant 0.35$ (for this range of volume fractions the order parameter $S$ is less than 0.5 irrespective of the aspect ratio and we will show later that this corresponds to lack of alignment-induced velocity correlation) underestimates the measurements of $s \dot{\gamma}$ in the numerical simulations for frictionless cylinders, irrespective of the aspect ratio, while it matches the numerical results for frictionless spheres (Fig. 2). The reason is that the geometry of the collision between two cylinders can induce rotation even in the absence of friction; hence, translational kinetic energy can be transformed into rotational kinetic energy. From the point of view of the balance of translational kinetic energy, this corresponds to an additional loss. A phenomenological attempt to take this effect into account without actually solving for the rotational kinetic energy balance (and therefore without introducing the rotational granular temperature) is by introducing an effective coefficient of restitution in Eq. (7). Inverting Eq. (7) with Eq. (6), we obtain

$$
e_{\mathrm{eff}}=\left(1-\frac{\pi^{1 / 2} s \dot{\gamma} d_{v}}{12 \rho_{p} v^{2} g_{0} T^{3 / 2}}\right)^{1 / 2},
$$

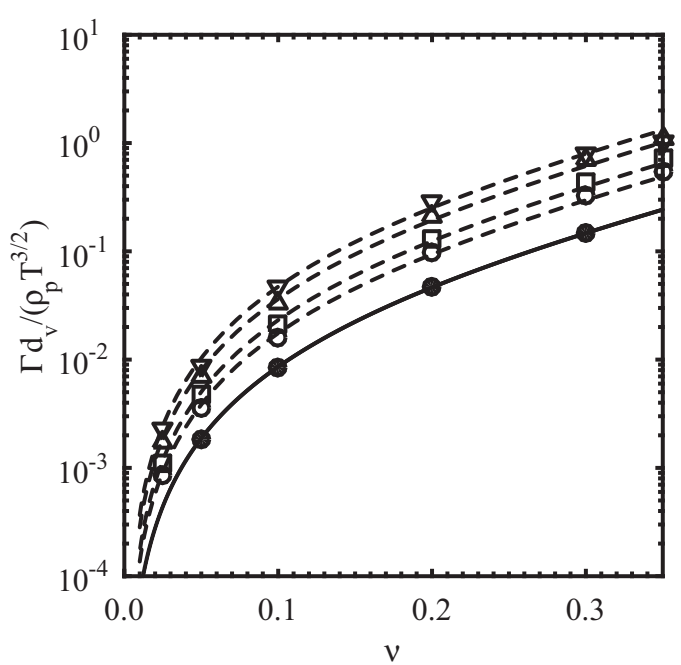

(a)

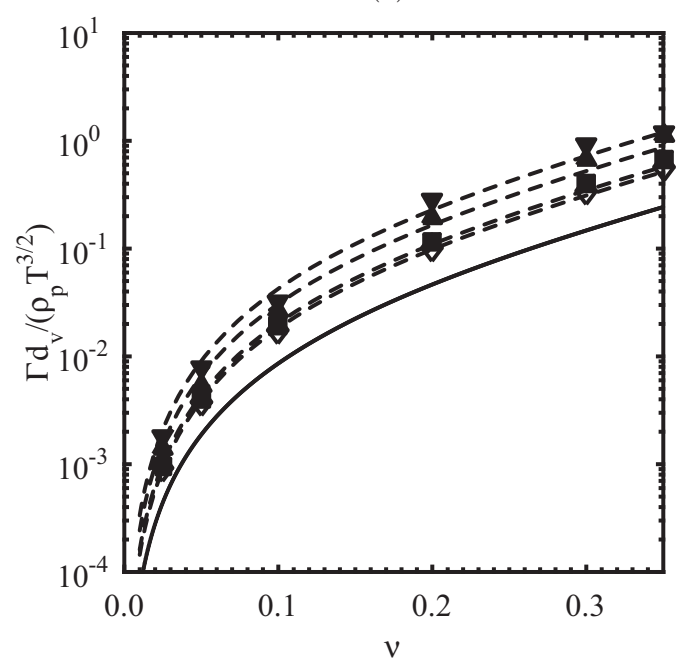

(b)

FIG. 2. Numerical (symbols) and theoretical (dashed lines) (using the effective coefficient of restitution) dimensionless collisional dissipation rate versus solid volume fraction for (a) elongated and (b) flat cylinders when $v \leqslant 0.35$ and $l / d=0.17$ (closed down triangles), $l / d=0.25$ (closed up triangles), $l / d=0.5$ (closed squares), $l / d=$ 0.8 (open diamonds), $l / d=1$ (open circles), $l / d=2$ (open squares), $l / d=4$ (open up triangles), and $l / d=6$ (open down triangles). Also shown are the numerical (closed circles) (after [20]) and theoretical (solid line) results for simple shearing of frictionless spheres having $e=0.95$.

which can be fitted to the numerical measurements. With this, the agreement of the expression of kinetic theory with the numerical measurements of the collisional dissipation rate are satisfactory for both elongated and flat cylinders (dashed lines in Fig. 2). The values of the effective coefficient of restitution normalized to the nominal value $e=0.95$ for different aspect ratios is reported in Fig. 3. It peaks when $l / d$ is equal to 1 and decreases with increasing flatness or elongation of the cylinders.

When the solid volume fraction exceeds the value at freezing point ( 0.49 for spheres), correlation in fluctuation velocities develops for inelastic spheres [11]; because of this correlation, 


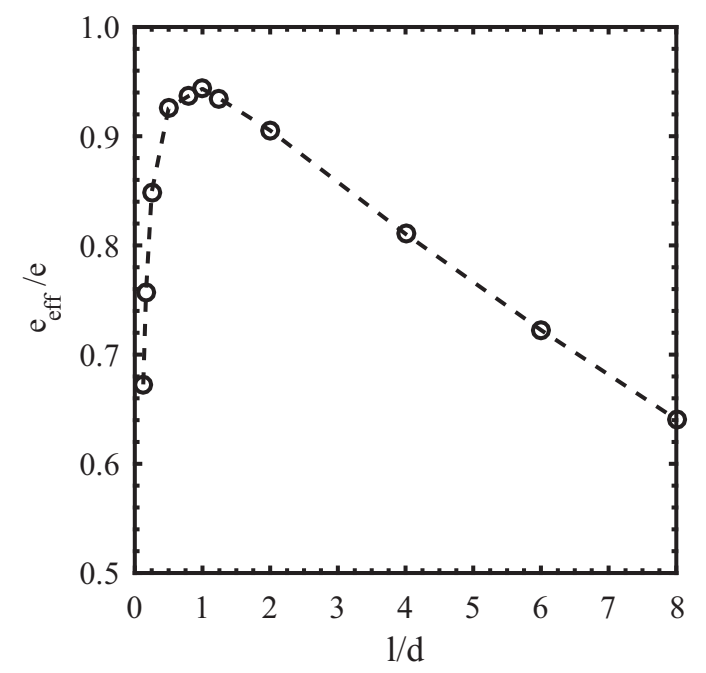

FIG. 3. Normalized effective coefficient of restitution versus aspect ratio.

not every fluctuation in the velocity of the single particle results in a collision and therefore $T^{1 / 2} / d_{v}$ (which measures the frequency of the fluctuations of the single particle) is no longer a good measure of the frequency of colliding events. In this situation, the collisional dissipation rate of Eq. (7) overestimates the results of numerical simulations. Similarly, Eq. (7) with $e_{\mathrm{eff}}$ instead of $e$ overestimates the numerical measurements at large volume fractions also in the case of cylinders (Fig. 4). Jenkins [13,21] has proposed to take into account this effect, for spheres, by introducing a correlation length $L$ instead of $d_{v}$ in Eq. (7). The quantity $L$ measures the size of a typical cluster of particles moving in a correlated fashion; hence, $T^{1 / 2} / L$ represents a reduced collision frequency that should apply at solid volume fractions exceeding the freezing point. If we use $L$ instead of $d_{v}$ and invert Eq. (7), we obtain, with Eq. (6),

$$
L=\frac{12}{\pi^{1 / 2}} v^{2} g_{0}\left(1-e_{\mathrm{eff}}^{2}\right) \frac{\rho_{p} T^{3 / 2}}{s \dot{\gamma}} .
$$

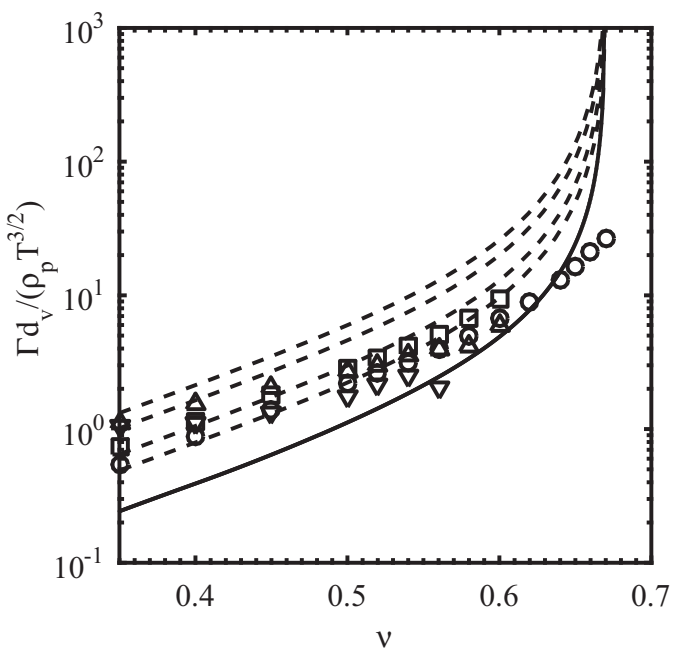

FIG. 4. Same as in Fig. 2(a), but for $v \geqslant 0.35$.

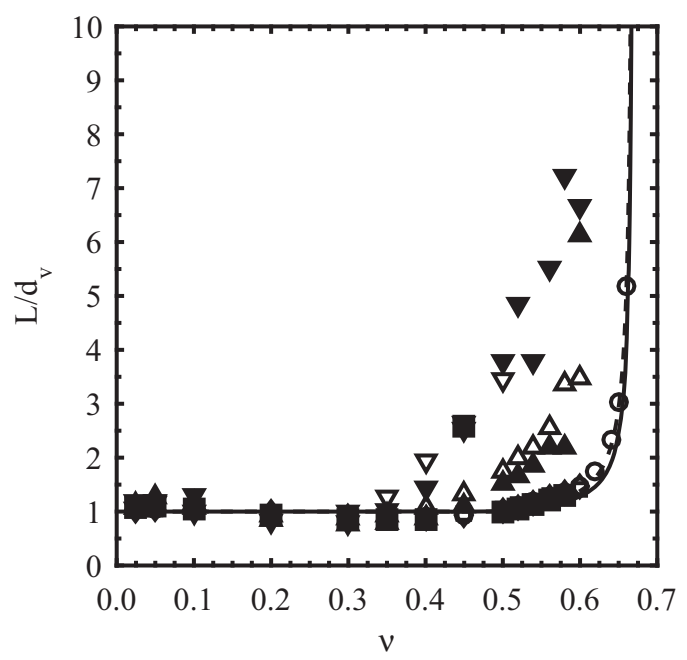

FIG. 5. Numerical (same symbols as in Fig. 2) dimensionless correlation length as a function of the solid volume fraction. The solid and dashed lines represent Eq. (10) when $e_{\text {eff }}=0.9$ (i.e., $l / d=1$ ) and 0.86 (i.e., $l / d=2$ ), respectively.

Equation (9) indicates that the correlation length can be estimated from the numerical measurements of solid volume fraction, granular temperature, and shear stress, with $e_{\text {eff }}$ taken from Fig. 3. The results of the numerical simulations are reported against $v$ in Fig. 5. It can be noticed that the data collapse onto a single curve for $l / d$ in the range $0.5-2$ (that is, when the order parameter is always much lower than 1, indicating that the cylinders are orientationally disordered [8]), with a sharp increase in the proximity of $v=0.67$, i.e., where also the stresses diverge. We have found that the expression

$$
\frac{L_{e}}{d_{v}}=\frac{26\left(1-e_{\text {eff }}\right)}{15} \frac{\max (v-0.49,0)}{0.674-v}+1
$$

reproduces well the numerical results for that range of aspect ratios (Fig. 5). Equation (10) matches the expression suggested in [16] and valid for simple shearing of frictionless and frictional spheres [19], with the important difference that the singularity is at $v=0.674$ (slightly larger than 0.67 , i.e., the singularity in the stresses for frictionless cylinders) instead of 0.64 (slightly larger than 0.636 , i.e., the singularity in the stresses for frictionless spheres [19]). In Eq. (10) we have used the subscript $e$ to emphasize that that expression for the correlation length is meant to take into account the velocity correlation induced by particle inelasticity.

When the aspect ratio is less than 0.5 or greater than 2 , instead, the numerical measurements of correlation length significantly depart from Eq. (10) and $L$ is greater than one diameter even at solid volume fractions less than 0.49 (Fig. 5). Given that, in that range of aspect ratio, the order parameter $S$ has the largest values [8], Fig. 5 suggests that both particle alignment and inelasticity concur to induce velocity correlation. When the numerical measurements of $L$ are plotted against the order parameter $S$ for $l / d<0.5$ and $l / d>2$ (Fig. 6), the data collapse onto a single curve (but for one outlier), which is well fitted by

$$
\frac{L}{L_{e}}=\frac{\max (S-0.5,0)}{1-S}+1 .
$$




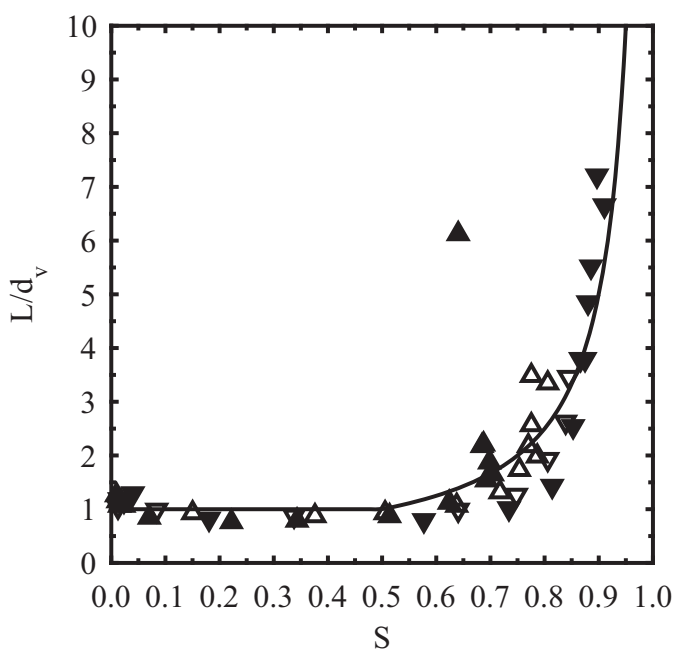

FIG. 6. Numerical (same symbols as in Fig. 2) dimensionless correlation length as a function of the order parameter. The solid line represents Eq. (11).

Figure 6 confirms, as anticipated, that no alignment-induced velocity correlation is present when $S$ is less than 0.5 . Equations (11) and (10), used in

$$
\Gamma=\rho_{p} \frac{12}{\pi^{1 / 2}} v^{2} g_{0}\left(1-e_{\mathrm{eff}}^{2}\right) \frac{T^{3 / 2}}{L},
$$

completely define the collisional dissipation rate for simple shearing of inelastic frictionless cylinders.

\section{CONCLUSION}

Following our previous work on the phenomenological modification of the constitutive relations of kinetic theory to describe stresses in granular liquid crystals composed of inelastic frictionless cylinders, we have here focused on the constitutive relation for the collisional dissipation rate. The latter is crucial in the balance of the fluctuation kinetic energy that governs the evolution of the intensity of the particle velocity fluctuations. We have made use of discrete-element simulations of simple shear flows at constant volume of frictionless cylinders at different particle aspect ratios and solid volume fractions. We have measured the work done by the shear stress in the numerical simulations, which, in simple shearing, exactly balances the collisional dissipation rate. This has allowed us to test the expression of kinetic theory and notice that, for solid volume fraction less than 0.35 , the collisional dissipation rate of classic kinetic theory works well, provided we adopt an effective coefficient of restitution that is less than the nominal coefficient of normal restitution that characterizes the particles in the simulations. The fitted value of the effective coefficient of restitution depends on the particle aspect ratio and has a maximum when the cylinder height equals its length. We have explained this observation by arguing that the geometry of the particles induces rotation in collisions even in the absence of friction, hence transforming ("dissipating" in a sense) translational kinetic energy into rotational kinetic energy. The effective coefficient of restitution models this additional pseudodissipation. At larger solid volume fractions, instead, the expression of classic kinetic theory, modified with the use of the effective coefficient of restitution, overestimates the measurements. The same observation, explained by the development of velocity correlation due to particle inelasticity, led to a phenomenological modification of classic kinetic theory in the case of spheres, with the introduction of a correlation length instead of the particle diameter in the dissipation rate. Assuming that this is the case also for cylinders, we have determined the dependence of the correlation length on the solid volume fraction by fitting the measurements. We have obtained that the expression valid for spheres holds also in the case of moderately flat or elongated cylinders. In the general case, however, the preferential alignment of the particle axis concurs with inelasticity in enhancing the velocity correlation, thus increasing the correlation length. We have derived a simple expression for the dependence of the correlation length on the degree of orientational order. The role of surface friction and particle stiffness on the constitutive relations and the solution of the continuum theory in more complicated flow configurations are left for future investigation.
[1] Y. Guo, C. Wassgren, W. Ketterhagen, B. Hancock, B. James, and J. Curtis, J. Fluid Mech. 713, 1 (2012).

[2] Y. Guo, C. Wassgren, B. Hancock, W. Ketterhagen, and J. Curtis, Phys. Fluids 25, 063304 (2013).

[3] M. Kodam, M. Bharadwaj, J. Curtis, B. Hancock, and C. Wassgren, Chem. Eng. Sci. 65, 5852 (2010).

[4] Y. Guo, C. Wassgren, W. Ketterhagen, B. Hancock, and J. Curtis, Powder Technol. 228, 193 (2012).

[5] T. Börzsönyi, B. Szabó, G. Törös, S. Wegner, J. Török, E. Somfai, T. Bien, and R. Stannarius, Phys. Rev. Lett. 108, 228302 (2012).

[6] H. Capart and L. Fraccarollo, Geophys. Res. Lett. 38, L20402 (2011).

[7] D. Berzi and L. Fraccarollo, Phys. Rev. Lett. 115, 194501 (2015).

[8] D. Berzi, N. Thai-Quang, Y. Guo, and J. Curtis, Phys. Rev. E 93, 040901(R) (2016).
[9] J. Jenkins and S. Savage, J. Fluid Mech. 130, 187 (1983).

[10] V. Garzó and J. W. Dufty, Phys. Rev. E 59, 5895 (1999).

[11] N. Mitarai and H. Nakanishi, Phys. Rev. E 75, 031305 (2007)

[12] V. Kumaran, J. Fluid Mech. 632, 145 (2009).

[13] J. T. Jenkins, Granular Matter 10, 47 (2007).

[14] J. Jenkins and D. Berzi, Granular Matter 12, 151 (2010).

[15] J. Jenkins and D. Berzi, Granular Matter 14, 79 (2012).

[16] D. Berzi, Acta Mech. 225, 2191 (2014).

[17] D. Vescovi, D. Berzi, P. Richard, and N. Brodu, Phys. Fluids 26, 053305 (2014).

[18] A. W. Lees and S. F. Edwards, J. Phys. C 5, 1921 (1972).

[19] D. Berzi and D. Vescovi, Phys. Fluids 27, 013302 (2015).

[20] S. Chialvo and S. Sundaresan, Phys. Fluids 25, 070603 (2013).

[21] J. T. Jenkins, Phys. Fluids 18, 103307 (2006). 